\title{
The risk of liquid wood degradation under the influence of marine factors
}

\author{
Mihaela Luminita Barhalescu ${ }^{1, *}$ Simona Ghita ${ }^{1}$, and Tudor-Cristian Petrescu ${ }^{2}$ \\ ${ }^{1}$ Constanta Maritime University; Naval Electromechanics Faculty, Genaral Engineering Sciences \\ Department, 104 Mircea cel Batran Street, 900663, Constanta, Romania, e-mail: \\ mihaela.barhalescu@cmu-edu.eu \\ ${ }^{2}$ Constanta Maritime University; Naval Electromechanics Faculty, Engineering Sciences Department \\ in Mechanical and Environment, 104 Mircea cel Batran Street, 900663, Constanta, Romania, e-mail: \\ simona.ghita@cmu-edu.eu \\ 3“"Gheorghe Asachi" Technical University of Iasi; Faculty of Civil Engineering and Building \\ Services, 67 Dimitrie Mangeron Street, 700050, Iaşi, România, e-mail: tudor.petrescu@tuiasi.ro
}

\begin{abstract}
A case study was performed, concerning the behavior and degradation of a polymeric biocomposite material - "liquid wood". This material is biodegradable and it is obtained from renewable resources. Three presentation forms - Arbofill Fichte, Arboform F45 and Arboblend V2, were subjected to the action of external factors present in a marine environment. The results pertaining to the change in the physico chemical properties of "liquid wood" when subjected to the action of seawater and seawater microorganisms, with significant - but nevertheless positive - consequences upon the environment. The material exhibits good performance after the surface and mass stabilization - due to water, $\mathrm{C}, \mathrm{Na}$, $\mathrm{Cl}$ and $\mathrm{O}$ absorption. As such - due to the emergence of a protective organic biofilm - growth of microorganisms significantly decreases and electric conductivity increases. This case study may be viewed as a starting point for subsequent studies of "liquid wood".
\end{abstract}

\section{Introduction}

After the signing of international agreements on pollution prevention, several directions have been drawn for this purpose [1-2]. These directions are: priority for research funding, with the aim of finding long-term solutions for the decrease of pollution, seeking alternative energy sources, the discovery of new materials obtained from renewable resources, which can substitute polluting materials such as ,plastics”.

There is a continuous search for new materials, particularly if they are from non-toxic ingredients, biodegradable and from renewable resources. Materials that can satisfy these exigencies are suited for specific applications, are highly desirable and represent a continuous and collective effort made by the human society to research and eventually put them to use [3].

\footnotetext{
* Corresponding author: simona.ghita@cmu-edu.eu
} 
One of these materials is commonly known as "liquid wood", which is a material obtained from renewable resources.

According to the products' technical specifications, ,liquid wood" is a composite biopolymer, based on a lignin matrix.

This material exhibits a series of specific properties, which recommend it as a possible solution for the replacement of ,plastics”. The „,eco-properties” of „liquid wood" are as follows: it is sourced from renewable raw materials, biodegradable under certain conditions and fireproof when reinforced with small quantities of other substances (ex.: Kevlar 3\%).

„Liquid wood" is manufactured in to three distinct forms: Arboform F45, Arbofill Fichte and Arboblend V2. They mostly differ by the other natural substances - apart from lignin - that may be found in the material (ex.: resins, wax, vegetable fibres). „Liquid wood" may be considered a relatively new material.

The main component of ,liquid wood" is lignin both in natural and modified state.

It is known that lignin provides hardness to wooden plants, it also has antibacterial properties etc. Thus, it can be said that, among other properties, lignin exhibits a system of defense against destructive external agents.

Experimentally it has been noticed that the wooden fibre does not exhibit any protection to fungal attack, therefore lignin is non-resistant to such attacks.

Taking into account that lignin is a part of all three forms of "liquid wood" (even though the forms themselves are different in composition, with varying percentages of lignin), it follows that, in various conditions, the behavior of these materials is sometimes similar, other times being different. As such, Arboblend V2 is difficult to tell apart from a "classic" polymer, as its surface is smooth, with a compact, white aspect. It is made of lignin $(99 \%)$ and natural additives. Arboform F45 is composed of a lignin matrix $(30 \%-$ $60 \%$ ), improved with a significant percentage of flax or hemp fibers (40\%-60\%). Reinforcing the material with natural fibers improves its mechanical properties, making it more rigid. Unlike other types of "liquid wood", Arbofill Fichte is made of $60 \%$ lignin, to which petrochemical polymers are added, such as polyethylene or polypropylene, mixed with natural fibers.

Ongoing research is focused on degradation factors and what is their influence upon the properties and behaviour of „liquid wood”.

As such, it is necessary to determine the behaviour of "liquid wood" in various environments, in order to find the variation of the materials' physio-chemical properties in these environments. Following the analysis of the obtained results, a range of conclusions may be drawn, which aim to point towards suitable applications for „liquid wood”, in accordance with the Protocols agreed upon at Kyoto and Rio de Janeiro.

\section{Equipment and culture media}

\subsection{Equipment used for the analysis of physical and electrical properties of liquid wood}

This article presents results related to studies carried out to establish the behaviour of „liquid wood” (Arboblend V2, Arboform F45 and Arbofil Fichte) in a marine environment, when the electrical properties vary. The results enabled drawing conclusions, related to the degradation and biodegradation of ,liquid wood", when subjected to specific marine environmental factors.

Identical samples were immersed in distilled water and in seawater for 90 days.

The mass was established with a Partner XA 160 analytical scale. By measuring the mass, the hydration of the samples was estimated, according to the following method: 
Hydration (water absorbed by the sample $)=(\mathrm{mf}-\mathrm{mi})$

where:

mi-initial sample mass (at moment 0 ), in $\mathrm{g}$

mf-sample mass after hydration (after 90 days), in $\mathrm{g}$

Establishing the conductivity was possible thanks to an apparatus of type Accumet XL30, Fisher Scientific.

The samples were initially weighed and then submerged in seawater and kept for 90 days at a $20^{\circ} \mathrm{C}$ temperature.

Then the samples were removed from seawater and were left to dry at constant temperature for two hours.

After drying, the samples' mass was again determined with the same analytical scale.

Early observations suggest that this was more than a simple hydration - other chemical compounds existant in seawater were absorbed as well.

As such, the focus of this article became the study of the behavior of ,liquid wood" when subjected to seawater exposure; of notable importance was the identification of chemical compounds absorbed by the material, as well as the way some microorganism colonies (ex. bacteria, fungi) developed from sea water.

Ascertaining the absorbtion of chemical compounds was done by direct observation with the help of scanning electron microscopy (SEM). The absorbed compounds were established by the EDX method and the XRF method.

For the same purpose of studying the degradation when subjected to seawater factors, the electrical properties of the samples were measured before and after the 90 days of immersion. The measurements were conducted by means of a broadband dielectric spectrometer type Novocontrol Concept 40 (GmbH Germania), with the frequency interval of $1-10^{6} \mathrm{~Hz}$, by placing the samples with a uniform thickness between two round electrodes with $1,0 \mathrm{~V}$ tension. The acquired results were used to plot some comparative graphs.

\subsection{Culture media used for microbiological detection}

For the study of the microorganisms action present in seawater - when in contact with „liquid wood" -, three types of specimens were selected (Arboblend V2, Arboform F45 and Arbofill Fichte), which were split in 4 pieces (3 samples and 1 control sample) and were placed in seawater at room temperature and exposed to natural light.

For the control samples, a surface view was performed, using an epifluorescence microscope (in the month of July). This proved useful, as it allowed the observation of the amount of microbiota (as a biofilm) on each type of sample at the beginning of the experiment. Colouring of the samples' surface was made using 1,8 $\mu \mathrm{L}$ SYBR Green $+1,8$ $\mu \mathrm{L}$ IP (propidium iodide) solution [4,5].

After 40 days of incubation (in the month of September) in a marine medium, all three samples were washed with distilled water $10 \mathrm{~mL}$. Following this, the water with organic content was filtered through a Millipore filter (filter membranes, nitrocellulose MF membrane, pore size $0.22 \mu \mathrm{m}$, diam. $47 \mathrm{~mm}$ ) in a vacuum system and the organic content has been put in a Compact Dry YM and Envirocheck bacteria/yeasts culture mediums.

Two types of culture medium were employed: Compact Dry YM and Envirocheck bacteria/yeasts.

\subsubsection{The Compact Dry YM}

The Compact Dry YM culture mediums allowed the separation of inferior fungi (yeasts and moulds), through different coloration. The medium contains a chromogenic X-Phos substrate, which colours a significant number of yeasts in blue-green. The moulds form 
fuzzy colonies, spotting a characteristic colour. Antibiotics suppress the development of bacteria and promote the tridimensional development of yeasts and molds.

The Compact Dry medium brings together the characteristics and the advantages of traditional mediums with dehydrated film mediums.

The incubation period ranges from 3 to 7 days at $25-30^{\circ} \mathrm{C}$. An incubation temperature of $29^{\circ} \mathrm{C}$ was used for the purpose of the experiments detailed in this paper.

\subsubsection{The Microbiologic Envirocheck Contact TVC}

The Microbiologic Envirocheck Contact TVC test spectrum is utilized for the quantitative detection of microorganisms on liquid surfaces, which are covered with various nutrient environments. After sampling and incubation, the results from side A (total number of bacteria) and side B (fungi highlighting) allow the establishment of parameters sourced from the working plate. These working plates are available for the following parameters:

- Fungi detection (red side)

- Detection of total number of bacterial colonies (white side)

In other words,

- Envirocheck Contact is a tube which contains a lamella with culture environments on both sides: on one of the sides there is a culture medium for bacteria development, which contains agar and $0.05 \%$ Triphenyl Tetrazolium Chloride (TTC). This side is used to enable bacteria growth, which is indicated by the formation of red-coloured colonies, with an incubation time of $24-48 \mathrm{~h}$.

- On the other side, there is another culture medium which is used for yeasts and moulds, which grow at $27-30^{\circ} \mathrm{C}$ for $2-7$ days.

The growth of fungi on the red surface has been taken into consideration.

Furthermore, after 40 days, besides the cultivation of samples on selective media, observations were made on the surface of every sample with the epifluorescence microscope using SG/IP staining, in the same manner that was done for the control sample.

A blue filter with $\lambda=450-480 \mathrm{~nm}$ was used.

Before colouring, each piece was washed with distilled water to remove the excess of organic matter and to be able to observe only the microbiota (as a biofilm) that had developed for one and a half months.

After three months of incubation in seawater at room temperature, all three samples are subjected to the same protocols used at 40 days after incubation.

\section{Results and discussion}

\subsection{Determination of chemical compounds absorbed by liquid wood and electrical properties after immersion in marine environment}

Due to the differences between the samples immersed in distilled water and those immersed in seawater, it was assumed that, besides water, other substances found in seawater were absorbed.

The estimation of absorbed salts mass was made with the following algorithm and is given in Table 1:

$$
\mathrm{m}_{\mathrm{s}}=\mathrm{m}_{\mathrm{f}}-\mathrm{m}_{\mathrm{i}}-\mathrm{m}_{\mathrm{a}}
$$

where:

$\mathrm{m}_{\mathrm{f}}$-final mass of the sample 
$\mathrm{m}_{\mathrm{i}}$-initial mass of the sample

$\mathrm{m}_{\mathrm{a}}$-estimated mass of embedded water

Table 1. Formatting sections, subsections and subsubsections

\begin{tabular}{|c|c|c|c|c|}
\hline Material & $\mathrm{m}_{\mathrm{i}}(\mathrm{g})$ & $\mathrm{m}_{\mathrm{f}}(\mathrm{g})$ & $\mathrm{m}_{\mathrm{a}}(\mathrm{g})$ & $\mathrm{m}_{\mathrm{s}}(\mathrm{g})$ \\
\hline Arboblend V2 & 1.500 & 1.922 & 1.593 & 0.329 \\
\hline Arboform F45 & 2.100 & 2.564 & 2.332 & 0.232 \\
\hline Arbofill Fichte & 1.600 & 1.864 & 1.740 & 0.124 \\
\hline
\end{tabular}

In order to test the validity of the theory of salt absorption, a series of specific physical tests were carried out. The absorbtion of chemical compounds in a transversal section made in a sample, can be observed in the images from Figure $1(\mathrm{a}, \mathrm{b}, \mathrm{c})$, which were obtained with the help of scanning electron microscopy (SEM). The absorbed compounds were established by the EDX method and the XRF method.

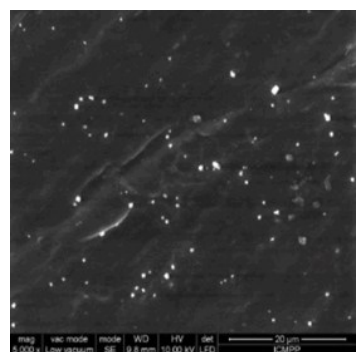

a

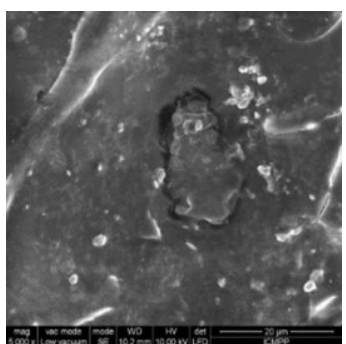

b

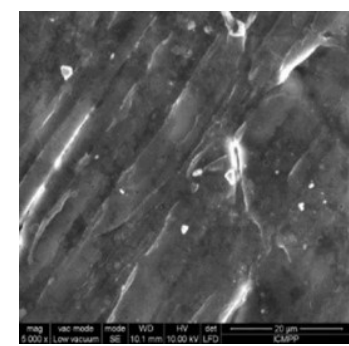

c

Fig.1. The surface images of a transversal section obtained through SEM, in which embedded crystals in Arboblend (a), Arboform (b) and Arbofill (c) can be observed

From data analysis, it can be noticed that the material that absorbs the most substances besides water is Arboblend. This is why the EDX method was used - it helps to determine what substances have been absorbed. The obtained results can be examined in Figure 2 and Table 2.
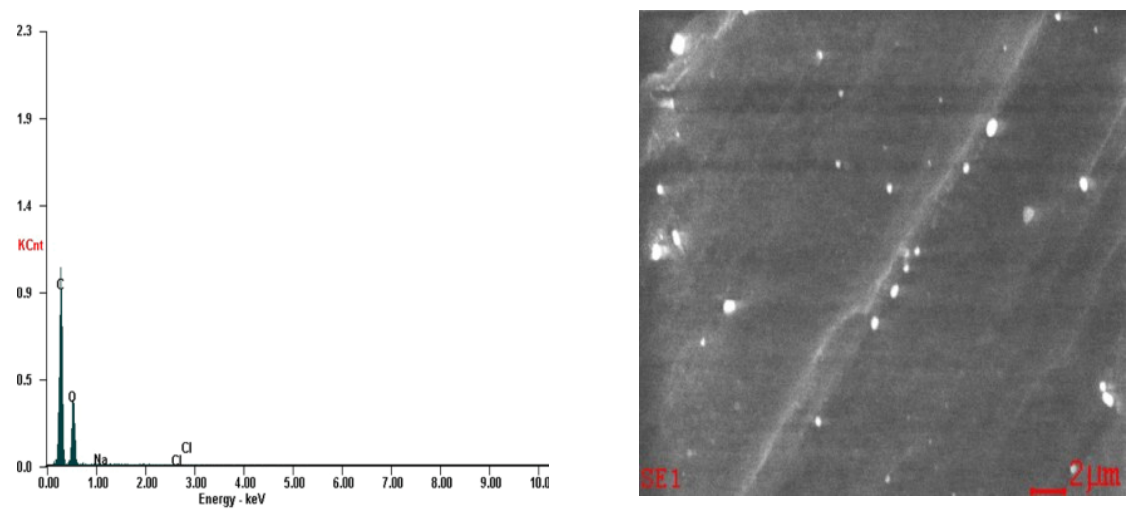

Fig.2. Substances absorbed by Arboblend 
Table 2. Substances with associated percentages embedded in Arboblend V2, following a 90-day immersion in seawater

\begin{tabular}{|c|c|c|}
\hline Element & $\mathrm{Wt} \%$ & $\mathrm{At} \%$ \\
\hline $\mathrm{CK}$ & 70.04 & 75.84 \\
\hline $\mathrm{OK}$ & 29.35 & 23.86 \\
\hline $\mathrm{NaK}$ & 00.38 & 00.21 \\
\hline $\mathrm{ClK}$ & 00.23 & 00.09 \\
\hline Matrix & Correction & $\mathrm{ZAF}$ \\
\hline
\end{tabular}

The quantities of embedded substances are highlighted with the help of XRF method (see Figure 3 a-e).

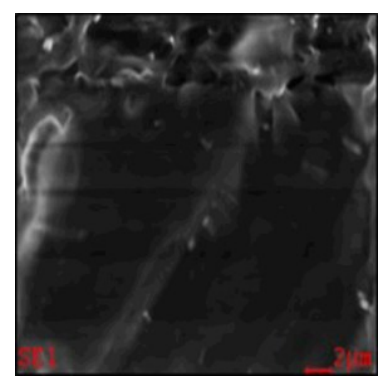

a

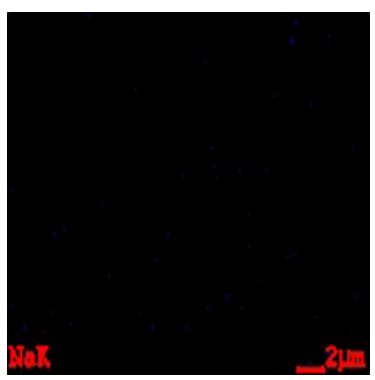

$\mathrm{c}$

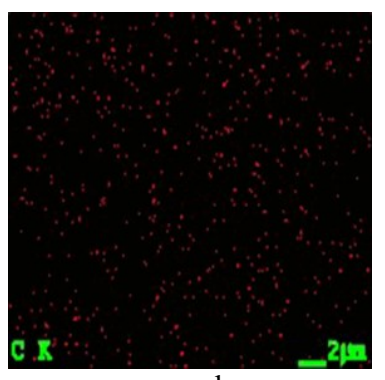

b

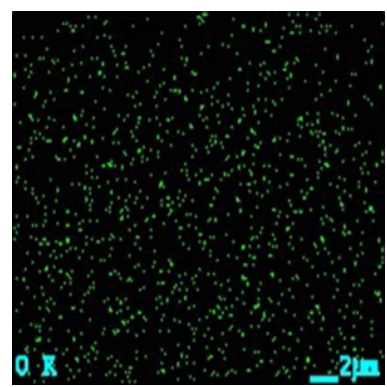

d

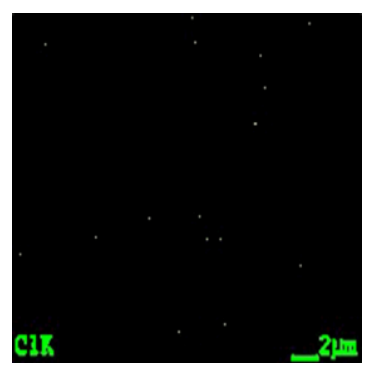

e

Fig.3. Substances embedded in Arboblend sample: a - "witness" sample; b - carbon; c-sodium; doxygen; e - chlorine 
The computation of electric properties such as relative permitivity er (Figure $4 \mathrm{a}-\mathrm{b}$ ), loss factor $\operatorname{tg} \delta$ (Figure 5, a-b) and conductivity $\sigma$ (Figure 6, a-b), permitted to observe the variation of these quantities, after 90 -day immersion in seawater.

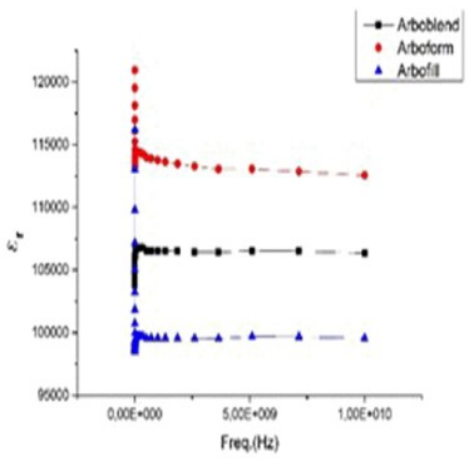

a

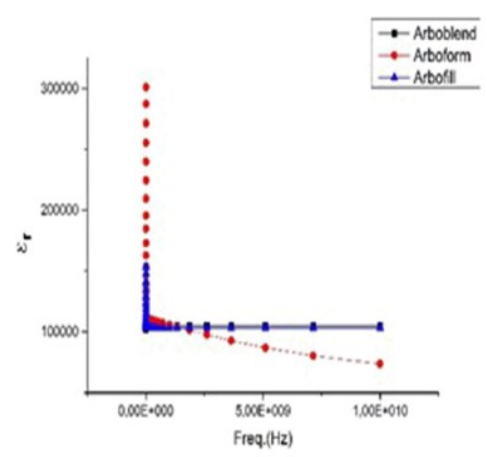

$\mathrm{b}$

Fig. 4. Relative permittivity \&r, a - before immersion, $b$ - after immersion in seawater

In figures $4(a-b)$ it can be observed that the relative permittivity changes; in the cases of Arboblend V2 and Arbofill Fichte, it becomes stable around the value of 105 and for Arboform F45, the value tends to drop below 105. It can be assumed that, for certain frequency values (also in the case of Arboform F45), the relative permittivity will tend towards a nil value [6].

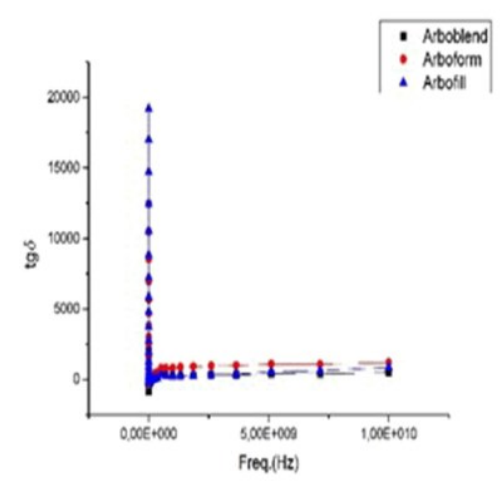

a

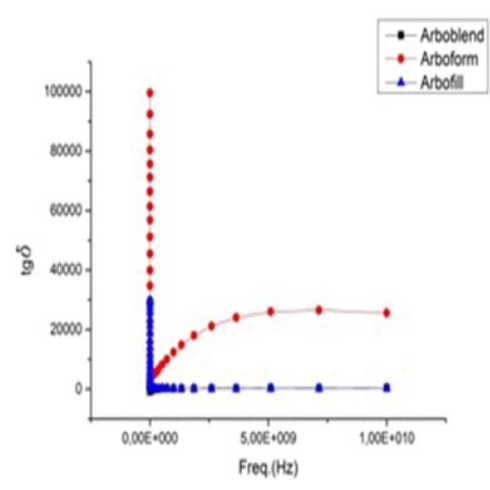

b

Fig. 5. Loss factor $\operatorname{tg} \delta$, $a$ - before immersion, $b$ - after immersion in seawater

In figures 5 (a-b), it can be observed that the loss factor doesn't modify in the cases of Arboblend V2 and Arbofill Fichte, but there is an increase of the value corresponding to Arboform F45, up to approximately $\left(2 \cdot 10^{4}\right)$ [7].

It can be observed that, for Arbofill Fichte and Arboblend V2, the graphs in figure 6a overlap, this meaning that the conductivity of the two materials varies identically before degradation. This aspect may be linked to the lignin percentages in the two materials. 


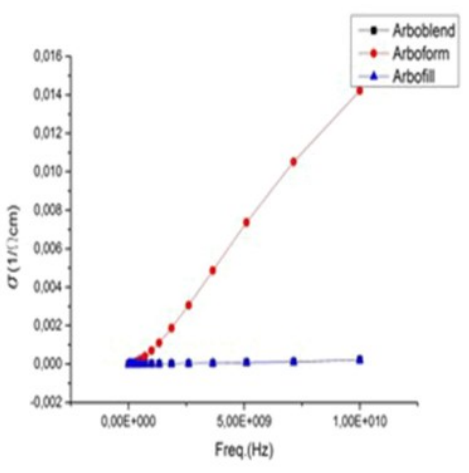

a

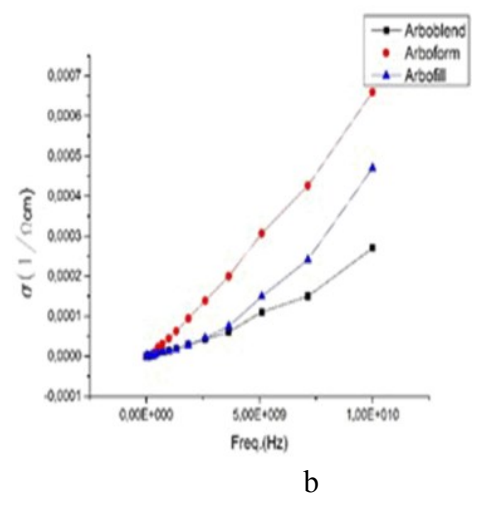

Fig. 6. Conductivity $\sigma$, a - before immersion, $b$ - after immersion in seawater

In figures $6(\mathrm{a}-\mathrm{b})$ it can be observed that conductivity increases in the case of Arboblend V2 and Arbofill Fichte, but decreases in the case of Arboform F45.

From the analysis of the three pairs of comparative graphs (figures $4,5,6$ ), it can be stated that all three materials undergo modifications regarding their electrical properties. In the case of Arboblend V2, electric permittivity decreases, the loss factor does not change and conductivity becomes nil. In the case of Arbofill Fichte, electric permittivity and the loss factor do not change, but its conductivity also becomes nil. Finally, in the case of Arboform F45, permittivity decreases, the loss factor increases and electric conductivity increases too. This may be explained by the polarization of the Arboform F45 sample, through the emergence of free electric loads.

These variations concerning the electrical parameters can be explained only by the absorption of water and other substances found in seawater.

The variation of electrical parameters for the three forms of "liquid wood" is an indicator of the degradation suffered by the materials when exposed to seawater; this statement is also backed up by the determinations regarding seawater (water + other substances) absorption.

\subsection{The evolution of microbiota on the liquid wood surfaces}

Regarding the behavior of "liquid wood" when acted upon by biological factors specific to maritime area, a determination of the multiplication factor for bacterial and fungi cultures has been attempted, through the microscopical observation of the colonies' evolution.

Furthermore, the fungi and bacterial species in the seawater that tend to biodegrade "liquid wood" were also identified. This issue was with tackled (and solved), by taking into account the specific fungi and bacteria that degrade lignin.

Because lignin is found in a high percentage in "liquid wood" (over $70 \%$ ), a comparative study has been made between the fungi and bacteria that degrade lignin and the fungi and bacteria that are present in the marine ecosystem. The common elements of these two categories are fungi from the genus Fusarium and from the genus Papulaspora.

After 7 days, the number of colonies grew in a different manner; in the case of Arboform F45, the colonies grew slower (34 colonies), but for Arboblend V2 and Arbofill Fichte the multiplying is progressive. This aspect can be observed in Figures $7(a, b, c)$ and $8(\mathrm{a}, \mathrm{b}, \mathrm{c})$. 


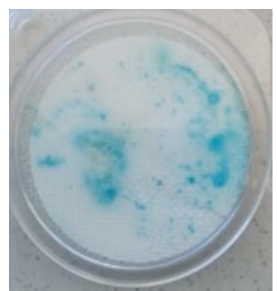

a

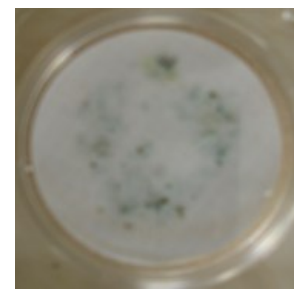

b

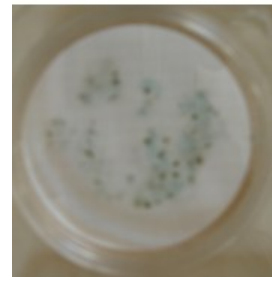

C

Fig. 7. Arbofill (a), Arboform (b) and Arboblend (c) samples obtained three days after incubation on the Compact Dry YM medium

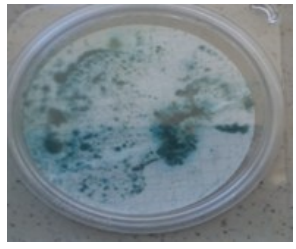

a

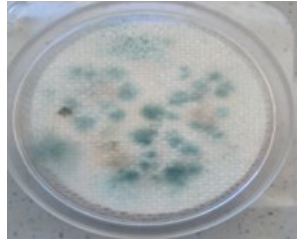

b

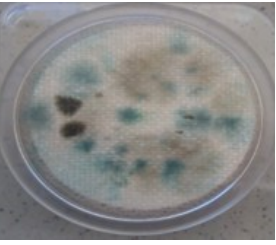

C

Fig. 8. Arbofill (a), Arboform (b) and Arboblend (c) samples obtained seven days after incubation on the Compact Dry YM medium

This type of culture (Compact Dry YM) is preferred, because it highlights the yeasts and the moulds - with the help of specific colouring- due to the X-Phos chromogenic substrate present in the environment and on the other hand through specific morphology aspects of the colonies (eg. moulds have a soft/fluffy texture of colonies). In the case of the aforementioned material samples, yeasts were particularly developed. To quantify the total number of yeasts and moulds was taken into account as being the equivalent of inferior fungi, to compare them with the other type of culture - Envirocheck Contact TVC, which inhibits rapid multiplication, but can allow them to increase in height.

For the Envirocheck Contact medium, the plates are covered with Bengal red cloramfenicol Agar (R). This medium is recommended for the selective isolation and quantification of inferior fungi (ex. yeasts and molds) from ecological materials and other biological products (ex. food). Cloramfenicol inhibits the development of supplementary bacteria on Bengal red. Bengal red is based on the size and height of mold colonies, so that the slow growth of fungi does not develop too many rapidly growing species.

After seven days, the number of fungal colonies increased and, at the same time, the surface on which the colonies were present at three days has expanded. This increase can be observed in the graph from Figure $9(a, b)$.

Based on this logical deduction, an algorithm which can model colony growth through mathematical modeling has been sought.

In Figures 10 and 11 the dynamic time-related development of fungi colonies on the surface of the three samples can be observed.

Based on data from the reference literature [8] and the extrapolation of data from the graphs of the development dynamics of fungi colonies (see figures 10,11) and empolying the OriginPro software, an empirical function that describe the abovementioned dynamics may be put forward.

The procedure was identical, regarding the development dynamics of fungi colonies on the second culture medium - Envirocheck Contact. The function is also exponential and has a similar form to the previous one (see Figure11).

By analysing Figures 10, 11, it can be observed that the development dynamics for the colonies of inferior fungi from the two types of cultures is similar, in the sense that for 
Arboblend V2 and Arbofill Fichte there is a quantifiable progression, whilst in Arboform F45 the growth is much slower, timewise. This aspect leads to the idea that, in the case of Arboform F45, there is a threshold for colony development, after which their number grows slower, possibly because of the lower lignin content than in the other two materials.

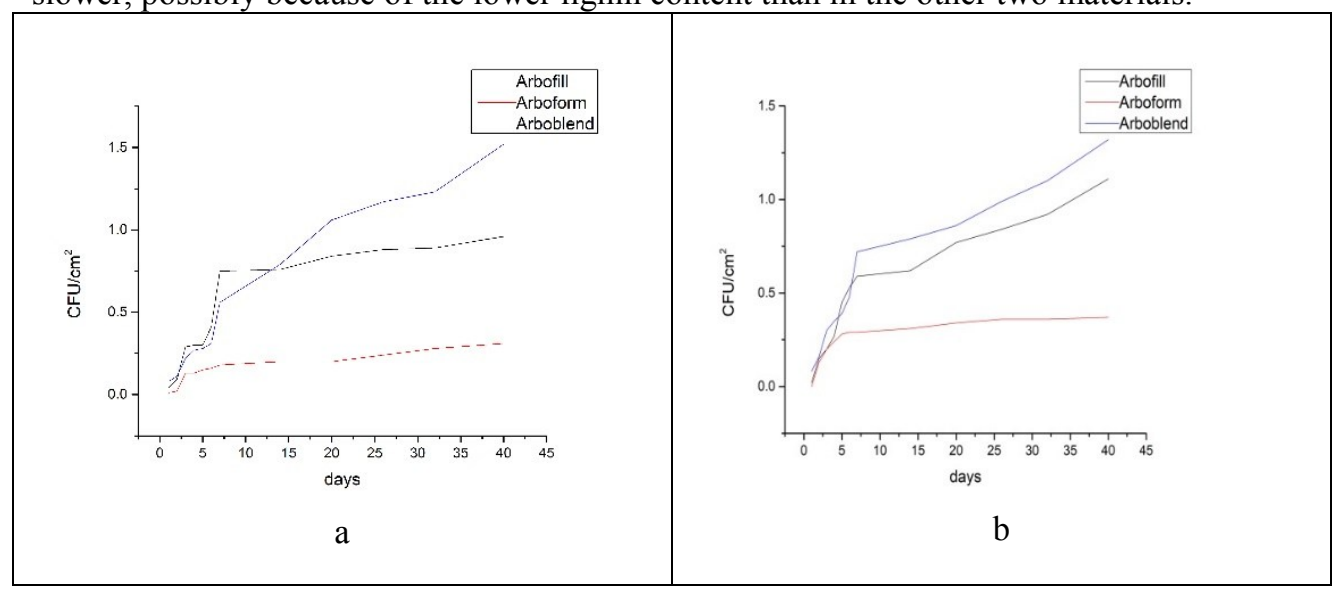

Fig.9. Variation in the number of colonies a) Compact Dry YM, b) Envirocheck Contact
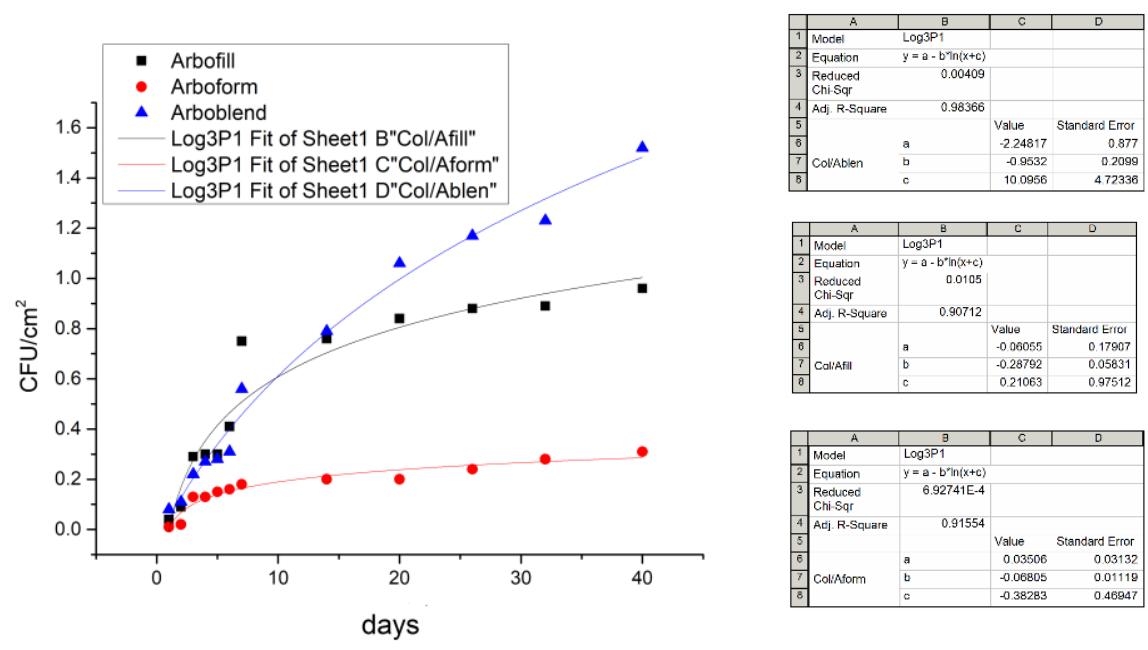

Fig. 10. The development dynamics of fungi colonies for the Compact Dry YM medium

In both mediums and for all types of liquid wood, the function is exponential and has approximately the same form; the only difference are the values of $\mathrm{a}, \mathrm{b}$ and c constants.

$$
y=a-b \cdot \ln (x+c)
$$

where:

- $\mathrm{y}$ - represents the number of fungi colonies on $\mathrm{cm} 2$

- $\mathrm{X}$ - represents time measured in days

Because fungi colonies exhibit a certain self-similarity, it is admitted that this process shows fractal properties, for the purpose of evaluating the multiplication dynamics of the colonies. This means that the dynamic variables that describe such a process are invariant to 
scale transformations. The mathematic apparatus of the invariant of any dynamic variable with respect to the scale resolution can be described as [9]:

$$
\mathrm{dQ} / \mathrm{d} \ln \lambda=\text { const. }
$$

The explanation of dynamic variables is:

$\mathrm{Q}=$ represents the multiplying rate of colonies

$\mathrm{Q}_{0}=$ initial rate of colonies

const. $=-\mathrm{b}$

$\lambda=\mathrm{x}+\mathrm{c}$

which ultimately represent the function:

$$
y=a-b^{*} \ln (x+c)
$$

The constants $\mathrm{a}, \mathrm{b}$ and $\mathrm{c}$ lock the scale resolution, in such a way that when a representation of $\mathrm{y}=\mathrm{y}(\mathrm{x})$ is made for the three types of liquid wood, basically three scale resolutions are obtained, each being defined by $a^{i}, b^{i}$ and $c^{i}$ parameters, where $i=1,2,3$.

In order to determine the multiplication mode of fungi colonies, the fractal dimension of colonies' multiplication dynamics is computed. The „box counting” method found in MatLab has been employed [10].
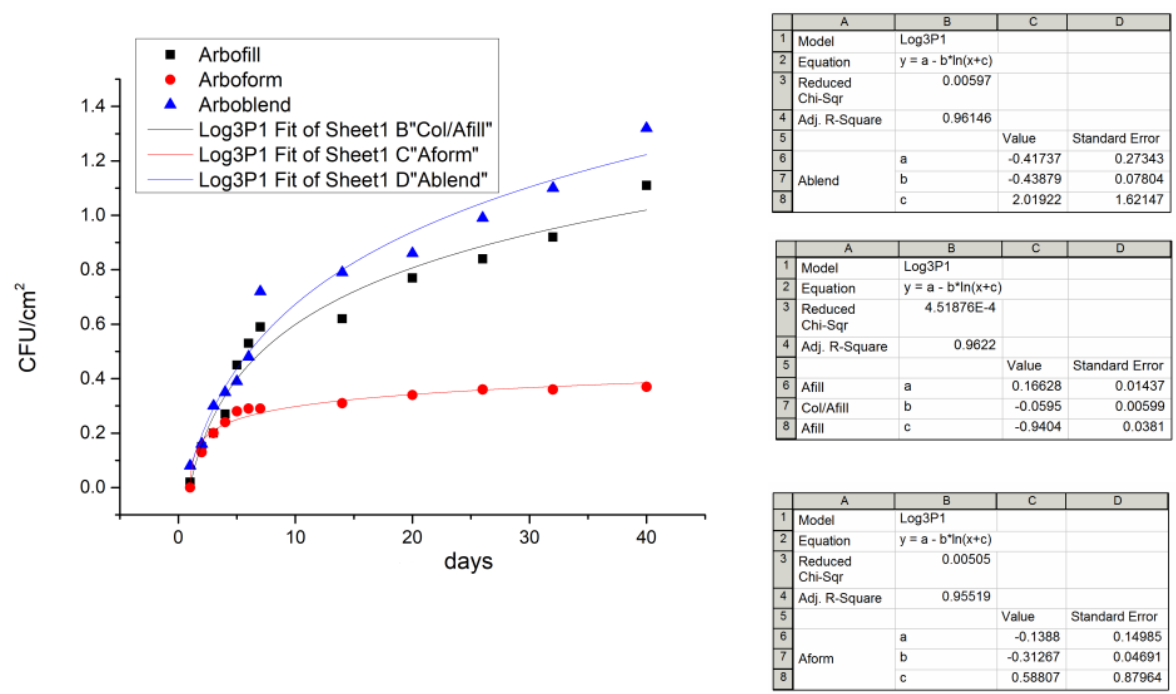

Fig. 11. The development dynamics of fungi colonies for the Envirocheck Contact medium

Following the computations, two graphs were obtained (Figures 12 and 13), which determine the fractal dimension $\mathrm{D}_{\mathrm{F}}$ for both study cases: Compact Dry $\mathrm{YM}$ and Envirocheck Contact.

From the study of the two graphs (Figures 12,13), it can be observed that the fractal dimension $\mathrm{D}_{\mathrm{F}}<2$, thus the dynamics of colonies' multiplication mainly occurs on the surface of the mediums.

The multiplication speed in the second case is greater than in the first case.

An explanation of the multiplication of the colonies on the surface of the mediums would be the fact that they are somewhat independent from one another and the surface development explains the tendency for the direct searching of food sources, in this case the lignin extant in the liquid wood.

It may be assumed that a total covering of the mediums' surface will occur, as well as a multiplication through vertical superposition. 


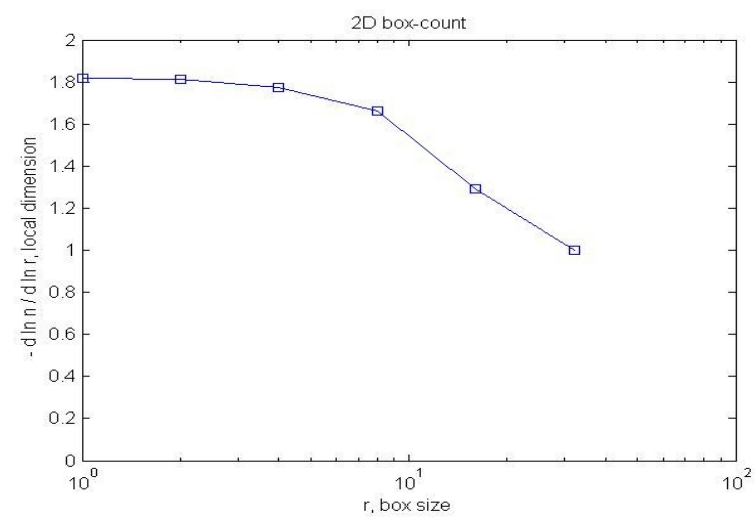

Fig. 12. Fractal dimension for Compact Dry YM

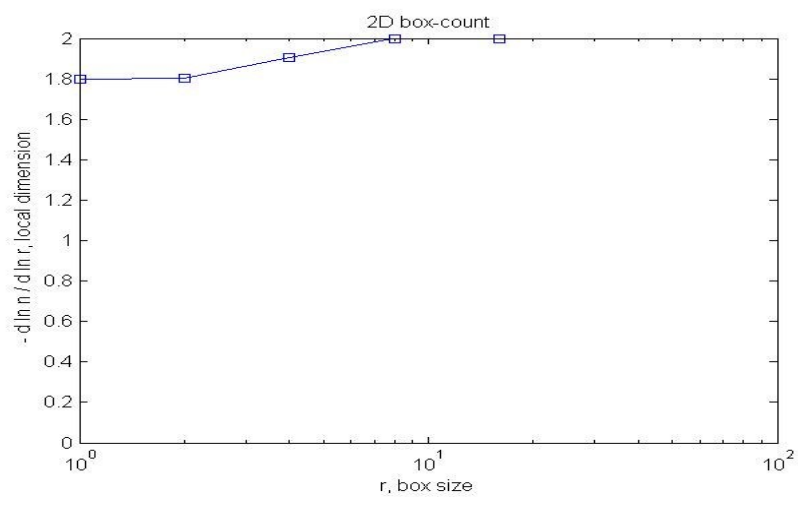

Fig. 13. Fractal dimension for Envirocheck Contact

We can estimate the moment when the total covering of the mediums' surface will occur by taking into account the dimensions of the samples $-16.9 \mathrm{~cm}^{2}$ and $10 \mathrm{~cm}^{2}$, respectively and the dimensions of fungi colonies $-1-3 \mu \mathrm{m}$ and $5-8 \mu \mathrm{m}$, respectively. begins.

It can be assumed that at that particular moment, the degradation of liquid wood

\subsection{Specific considerations regarding the evolution of biofilm formation}

At the begining of the experiment, observations were made on the epifluorescence microscope regarding the organic accumulation on the three studied surfaces. In this respect, we follow the evolution of the biofilm composed of heterotrophic bacteria, unicellular algae and cyanobacteria [11].

For the Arbofill Fichte sample with non-homogenous surface and numerous striations, the presence of homogeneously distributed and potentially quantifiable bacterial cells was observed. However, the presence of striations may hamper the visibility of cells on some microscopic fields (Figure 14 a).

For the Arboform F45 sample, non-homogenous surface, organic particles and a higher microbial and algae count have been identified, when compared to the previous sample (Arbofill Fichte) (Figure 14 b). 
For the Arboblend V2 sample with a homogenous surface, the presence of quantifiable and evenly distributed bacterial cells can be identified (Figure $14 \mathrm{c}$ ).

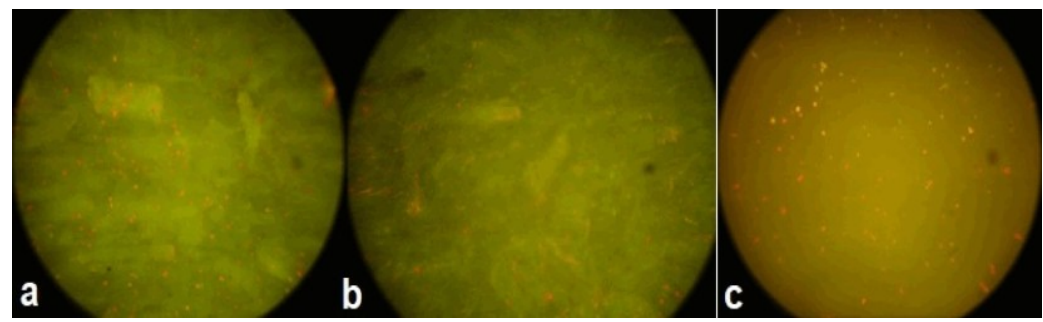

Fig. 14. Microscopic aspect of Arbofill, Arboform, arboblend sample surface at the beginning of the experiment (x1000 ob)

After 40 days, besides the cultivation of samples on selective media, observations were made on the surface of every sample under the epifluorescence microscope, using SG/IP staining, as in the case of control sample.

A blue filter with $\lambda=450-480 \mathrm{~nm}$ (Figure 15) was used.

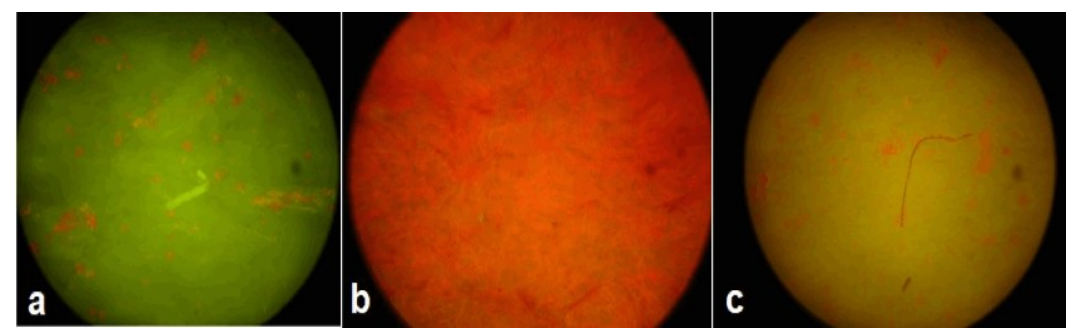

Fig. 15. Microscopic aspect of Arbofill sample (x1000 ob)

Before colouring, each piece was washed with distilled water, in order to remove organic matter in excess and then for one and a half months take observations of the microbiota attached to the surface.

On the Arbofill Fichte sample (Figure 15 a), some solitary unicellular green algae and cianobacteria, an homogenic distribution of microbiota composition, with a visible surface may be observed; on the Arboform F45 sample (Figure $15 \mathrm{~b}$ ), an increased density of filamentous cianobacteria which cover the entire surface of the sample may be scrutinized and on the Arboblend V2 sample (Figure 15 c) filamentous algae and organic matter are present in significant quantities on all microscopic fields.

A first conclusion can be drawn, namely, the development of bacteria and algae in the case of Arboform F45, determined a slower development of surface fungi, as compared to Arbofill Fichte and Arboblend V2 samples. The created biofilm depends especially on the characteristics of the contact surface, including the organic content.

The material from the vegetal cell wall is composed of three important elements: celulose, ligning and hemicelulose. Lignin is extremely difficult to biodegrade and also reduces the bioavailability of the other constituents of the cellular wall.

The factors which influence the presence of marine fungi include: water temperature, salinity, water dynamism, favourable colonization layers, presence of propagula in the water, interspecific competition, pollution, the dissolved oxygen content of water. Some 
marine fungi which migrated to the sea from the land include species that live in sand grains.

Temperature is one of the most important factors that can influence fungi growth. Other relevant factors are carbon and nitrogen sources, as well as the $\mathrm{pH}$ level.

Furthermore, the role of fungi in delignification due to the production of oxidative extracellular enzymes, has been studied more intensively than in the case of bacteria.

The fungi category describes yeasts on the one hand and molds respectively (filamentous fungi, micromycetes). The difference between the two types of culture environments related to fungi presence on a selective culture medium is presented in Figure 16.

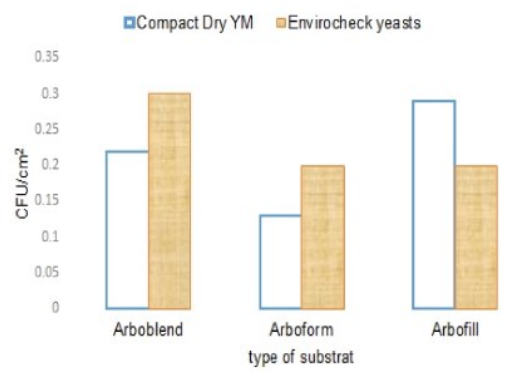

Fig. 16. Presence of microscopic fungi after 3 days of incubation

In the colonization of ,liquid wood" surfaces by fungi, for the two media small differences can be observed. Arboblend V2 and Arbofill Fichte are preffered by yeasts/molds, compared to Arboform F45. These differences result from the lignin composition/content of each of the surfaces.

Yeasts are commonplace in natural environments, being present in most natural habitats. From the ground and the air they can migrate into water, where some species can be found in at significant depths (over $1000 \mathrm{~m}$ depth).

The presence of molds in the aquatic environment is occasional in its nature and is mainly linked with the spore spreading of these species. The growth of molds in water depends on organic contents and aeration conditions.

Fungi presence determination on different surfaces of liquid wood was used as an indicator of their overall metabolic activity in the marine environment, which we considered as an incubation medium.

Fungi play an essential role in biodegradation, producing a series of extracellular and non-specific enzymes. Ligninolytic fungi can secrete extracellular oxidative enzymes that allow degradation of the lignin molecule [12].

Based on the enzymes it produces, fungi can degrade different types of polymers, not only lignin.

Considering the growth substrate the three types of liquid wood, it is not only composed of lignin, but also hemicellulose and cellulose, from where the difference in appearance of fungi comes.

Lignin is an amorphous biopolymer having an aromatic structure close to the aromatic molecular structure of environmental pollutants such as pesticides or even polycyclic aromatic hydrocarbons. Taking into account the degree of sensitivity and tolerance of the fungi, they are less sensitive than bacteria, the vast majority being aerobic organisms, having the potential to recover at normal temperatures, and the mycelium has an increased efficiency in capturing nutrients in the environment. 
Considering the marine environment as an oligotrophic environment, we can accept the idea that a moderate level of nitrogen is needed for fungi growth, although some fungi, especially those found decaying on wood, grow at low levels of nitrogen. Indeed, a low level of nitrogen is usually an initial condition for the degradation of lignin. Even so, low nitrogen levels are a limiting factor for celulose degradation speed.

Due to the molecular structure of the cell wall, fungi can absorb simple soluble nutrients. For cellular synthesis, fungi need to take the carbon from the environment, which is found in lignin (the basic structural unit being the Phenyl- propane (C6-C3) group). The carbon content in lignin is higher than in cellulose and hemicellulose, which results in the calorific value of lignin.

In our study, the fungus growth substrate on the Arboblend V2 and Arbofill Fichte surfaces was primarily lignin and secondarily the cellulose present in the algal component. In the Arboform F45 case ligninolytic activity may appear as a form of secondary metabolism of fungi, corroborated with the oligotrophic conditions of the environment (sea water).

\section{Conclusion}

From the data analysis, it can be observed that there is a maximum of water absorption, after which the samples cannot absorb any more water - a saturation threshold is reached.

"Liquid wood" is selective with regard to the absorbed substances.

It can be observed that, for Arboblend V2, the electrical properties undergo the least amount of changes.

At the same time, it can be said that for Arboform F45, there is a different variation of electric parameters than in the case of Arboblend V2 and Arbofill Fichte. This aspect can be explained by the different chemical composition of the materials [13]:

Arboform F45-70\% lignin plus other natural materials

Arboblend V2 - 99\% lignin plus other natural and synthetic materials

Arbofill Fichte $-60 \%$ lignin plus other natural and synthetic materials

Thus, being made only of natural substances, Arboform F45 behaves differently than the other forms of liquid wood and it may be stated that Arboform F45 "tries to oppose" degradation by modifying the electrical parameters in a different way than the other materials.

The created biofilm particularly depends on the characteristics of the contact surface, including organic content.

The biofilm includes the initial formation of the bacterial layer in which microorganisms are especially involved.

The surface of the Arboblend V2 sample determines a development of fungi and decomposing organic matter, whilst the surface of the Arboform F45 sample exhibits an attraction of bacteria and cianobacteria in high densities, without allowing a big growth of fungi. The Arbofill Fichte sample maintains the homogenity of microbiota due to its physico-chemical structure, thus the surface of the sample is visible.

The degradation speed of Arboform F45 is lower than in the case of Arbofill Fichte or Arboblend V2 [14].

The different types of biofilm on the experimental samples were due to physical and chemical characteristics of the liquid wood surface and also the content of the organic substance.

Based on performed analyses, as well as on obtained results, it can be stated that employing "liquid wood" - in any of its three presentation forms - on the coastal areas, a risk of accelerated degradation is present, due to the action of the marine environment. 
There is a risk that the degradation will significantly modify the physic - chemical properties of the material in a short amount of time, this aspect not being desirable.

Taking into account differential bacterial adhesion on the surface of the analyzed samples, a way of liquid wood surface treating by using methods that have a bactericidal effect, or modification of the hydrophilic character of the surface can be considered.

\section{References}

1. S. Ford, M. Despeisse, J. Clean. Prod., Additive manufacturing and sustainability: an exploratory study of the advantages and challenges, 137, 1573-1587 (2016)

2. Y. Li, D. Xu, C. Chen, X. X. Li, R. Jia, D. Zhang, W. Sand, F. Wang, T. Gu, A review. J. Mater. Sci. Technol, Anaerobic microbiologically influenced corrosion mechanismsinterpreted using bioenergetics and bioelectrochemistry., 34, 1713-1718 (2018)

3. D.R. Cooper, T.G. Gutowski, A review. J. Ind. Ecol., The environmental impacts of reuse, 21 (1), 38-56 (2015)

4. S. Ghiță, I. Ardelean, Microbes in Appl. Res.: Current Advances and Challenges, Correlation between direct viable count of Gram-negative bacteria and biological oxygen demand in marine microcosms polluted with gasoline, 65-69 (2012)

5. N. Acomi, S. Ghiţă, Ann. of DAAAM for 2012 \& Proc. of the $23^{\text {rd }}$, Using heat treatment of ballast water for killing marine microorganisms, 23(1), 1115-1118, (2012)

6. E. Puiu Costescu, S. Plavanescu Mazurchevici, C. Ursu, M. Agop, D. Vaideanu, Int. J. Mod. Manuf. Technol, The "Liquid wood" bahavior in electromagnetic field., 7(2), 48-52 (2015)

7. S. Plavanescu Mazurchevici, A. Salceanu, E. Puiu Costescu, D. Vaideanu, Bul. Inst. Politeh. Iaşi, Surface Behaviors of "Liquid Wood, Tomul LXI (LXV), 73-84 (2015)

8. S.A. Kharin, A.V. Kurakov, Microbiol., Characterization of the physiological state of fungi by dynamics of colony emergence on solid media, 82- 84 (2013)

9. R. Crișan Dabija, A. Zală, E. Hnatiuc, A. Agop, E. Puiu Costescu, D. Văideanu, I. Palamariuc, G. Jimborean, F. Nedeff, M.V. Ivan, Bul. Univ. Politeh. Bucur. Ser. A. Mat - Fiz., Fractality influences on a free gaussian "perturbation" in the hydrodinamic version of scale relativity theory. Possible implication in the biostructures dynamics, 281-292 (2017)

10. M. V. Ivan, A. Zală, A. Agop, E. Puiu, D. Văideanu, I. Palamaciuc, D.T. Iancu, R. CrișanDabija, Bul. Univ. Politeh. Bucur. Ser. A. Mat - Fiz., Several aspects about fractalitaty role in the dynamics of complex systems, 2017; 3, 235-246 (2017)

11. M. Hnatiuc, A. Sabau, B. Hnatiuc, S. Ghita, ModTech IOP Conf. Ser: Mater. Sci. and Eng, Comparative analysis of biofouling microorganisms after treatment with glidarc, 95, 012057 (2015)

12. C. Liers, T. Arnstadt, R. Ullrich, M. Hofrichter, FEMS Microbiol. Ecol., Patterns of lignin degradation and oxidative enzyme secretion by different woodand litter-colonizing basidiomycetes and ascomycetes grown on beech-wood, 78(1), 91-102 (2011)

13. E. Puiu Costescu, L. Leontie, M. Dumitraș, M. Asănduleasa, D. Văideanu, T.C. Petrescu, Bul. Inst. Politeh. Iaşi, The thermodynamic behavior of ,, liquid wood", 64 (68), 9-16 (2018)

14. D. Vaideanu, S. Plavanescu Mazurchevici, E. Puiu Costescu, D. Nedelcu, M. Agop, Ann. "Dunărea de Jos" Univ. Galaţi, Metal. and Mater. Sci, Fractal Logic Elements of Some Biodegradable Materials and Their Environmental Implications, Fasc. IX, 13-18 (2015) 